\title{
Role of step-shape structures and critical failure bubble volume in micro bubble constraint
}

\author{
ZHU XiaoGu, HUANG WeiFeng \& LI Yong* \\ State Key Laboratory of Tribology, Department of Precision Instruments and Mechanology, Tsinghua University, Beijing 100084, China
}

Received September 19, 2011; accepted April 19, 2012

\begin{abstract}
Micro fluid devices using micro bubbles have attracted much attention for their special advantages. In many cases, the bubbles must be constrained to a certain position so as to grow or shrink under control. In this paper, we discuss the effect of step-shape structures in constraining bubbles against the surface force, which plays a dominant role on the micro scale. We fabricate specimens with a step and electrodes and carry out electrolysis experiments in water to observe bubble growth. We observe the constraining effect of steps as well as failure phenomena. Based on numerical simulations and theoretical analysis, we introduce the critical failure bubble volume and present the analytic solution in a 2-D model. Using step-shape structures is an easy method of constraining bubbles within the critical failure volume..
\end{abstract}

micro bubbles, position constraint, step-shape structures, contact angle

Citation: Zhu X G, Huang W F, Li Y. Role of step-shape structures and critical failure bubble volume in micro bubble constraint. Chin Sci Bull, 2012, 57: 2941-2946, doi: 10.1007/s11434-012-5251-7

New micro devices are continually emerging as the development of microelectromechanical systems (MEMS) technology. However, new working principles always draw more attention because of better performance, especially in recent years. Micro gas bubbles have been used in a variety of new devices for their special advantages since thermal bubbles were successfully used in ink jet printer heads [1]. Micro bubbles have been used to compose micro pumps [2-4], micro valves [5,6] and micro mixers [7-10], promising no moving mass block, simple operation, miniature size, large actuation force and the ability to conform to different micro-channel cross sections [2]. In computer technology, micro bubbles are studied to create micro heat exchangers or cooling systems for electronic devices [11]. Besides micro fluidic systems, micro bubbles are also applied to conventional MEMS devices such as accelerometers, and have the advantages of no solid-proof mass, good frequency response and high sensitivity [12,13].

In many situations, micro bubbles are required to stay at

*Corresponding author (email: liyong@mail.tsinghua.edu.cn) a certain position so as to grow or shrink under control. Ryu et al. [3] found that the resonance frequency of their micro pump significantly changed with position offset of the bubble, thus weakening the actuation force. In the thermal bubble micro valve of Tsou et al. [5], a micro bubble is required to remain stably at the heating point throughout the growth period till the channel is blocked. Ahmed et al. [8,9] used bubbles trapped in channels in their micro mixers. Besides, in cell-related micro-scale research, gas bubbles in micro-fluidic channels may damage cells through the liquid-gas interface, which requires trapping and removing bubbles before they enter the main channel network [14]. Therefore, constraining micro bubbles is important for correlative micro-fluidic devices.

An efficient method to control liquid-gas distribution is to construct a special wettability pattern on the solid surface $[15,16]$. However, the wettability is often limited by materials and manufacturing processes. An alternative method to constrain bubbles is to construct special local structures, which can be more widely applied to micro devices. Ahmed et al. [8] designed sidewall grooves and a horse-shoe struc- 
ture [9] in the device channel to trap bubbles. Kang et al. [14] fabricated "well" structures along the channel to trap gas bubbles via buoyancy and hold their position via surface tension, and these hemispherical wells showed better performance than cylindrical ones in the experiments.

Various structures have been used to constrain micro bubbles. However, there is hardly any research on the constraining mechanism, thus hardly any theory for designing. At present, these structures are designed mostly by the experience and intuition of the authors. In this paper, we focus on the micro step-shape structure, a simple but typical structure in MEMS and micro-fluidic systems, to reveal some of its effect on constraining bubbles. We first conduct experiments to observe the constraining effect of steps; then, we perform theoretical analysis and numerical simulation to find the relationship between this constraining effect and correlative geometrical and physical parameters.

\section{Idea and experiments for constraining bub- bles with step-shape structures}

The natural wettability of a surface, limited by the materials and manufacturing processes, often goes against the demand for constraining bubbles. Electrolytic or thermal bubbles, for example, generated on the metal electrodes or heating resistors of strong wettability, will move to the surrounding surface of weaker wettability, rather than stably hold their initial positions. Under this unfavorable condition, structures of certain shapes may act as obstacles to bubble movement, so as to constrain the bubbles and control their positions.

\subsection{Idea of constraining with step-shape structures}

In equilibrium, gas-liquid interfaces at an ideally clean and inert flat solid surface are governed by Young's law:

$$
\gamma_{\mathrm{LS}}+\gamma_{\mathrm{LV}} \cos \theta=\gamma_{\mathrm{SV}}
$$

where $\theta$ is the equilibrium contact angle, which reflects the physical properties of the materials, and $\gamma_{\mathrm{LS}}, \gamma_{\mathrm{LV}}$ and $\gamma_{\mathrm{SV}}$ are the interfacial tensions between the liquid-solid, liquidvapor, and solid-vapor interfaces, respectively.

When the flat solid surface is composed of two or more domains with different contact angles, a bubble happening to lie on the borderline will have an asymmetric shape, as shown in Figure 1(a) where $\theta_{\mathrm{I}}>\theta_{\mathrm{II}}$. The resultant force of the surface tension on such an asymmetric bubble points to the side that has a bigger contact angle [14,17], thus inevitably pulling the bubble off the borderline, unless counterbalanced by other external forces.

With a sill or step-shape structure added to domain I near the borderline in Figure 1(b), a bubble of proper volume may stably hold its position on the borderline. In fact, due to the asymmetric structure, the resultant force of pressure on

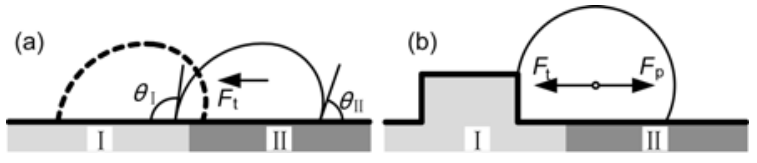

Figure 1 (a) A bubble transferring to the domain with a bigger contact angle; (b) a bubble held by a step.

the gas-liquid interface points away from the step and counterbalances the surface tension.

\subsection{Experimental apparatus and method}

Specimens with step-shape structures are designed with electrodes at the lower surfaces of the steps, as shown in Figure 2. These specimens are immersed in water and generate gas bubbles while electrified.

These specimens are fabricated by MEMS processes, which start with a silicon wafer. First, thermal oxidation is used to grow a 5000- $\AA$ silicon oxide insulation layer. Then, a lift-off method is used to sputter on and pattern a 400/300/900 $\AA \mathrm{Ti} / \mathrm{Pt} / \mathrm{Au}$ triple-layer to form comb-shape electrodes (Figure 2(b)). Finally, a photoresist layer is spun on, forming steps after exposure and development. We have chosen two different thicknesses for the photoresist layer, 1 and $15 \mu \mathrm{m}$, for our experiment.

The experiments are carried out in DI-water under atmospheric pressure at room temperature with a DC power supply. We observe the electrolysis reaction and record it with a digital microscope, as illustrated in Figure 3. At the beginning, the DC power supply voltage is set at $3 \mathrm{~V}$, and then is increased carefully until some gas bubbles appear and grow slowly and steadily enough for observation. The microscopic video starts from the beginning, and its recording frame rate is $25 \mathrm{~Hz}$.

\subsection{Experimental results}

We can see the constraining effect of steps in our experiments. During the electrolytic reactions using specimens with a $15-\mu \mathrm{m}$ photoresist layer, the electrolytic bubbles can stay at the metal surfaces of the electrodes even until they

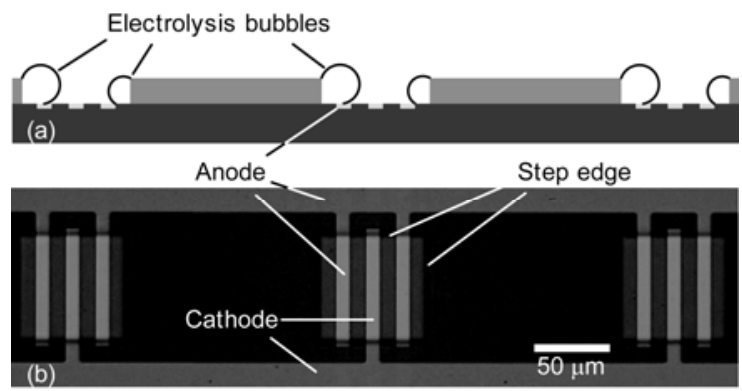

Figure 2 (a) A sketch for test specimen from cross section view; (b) a micrograph from top view. 


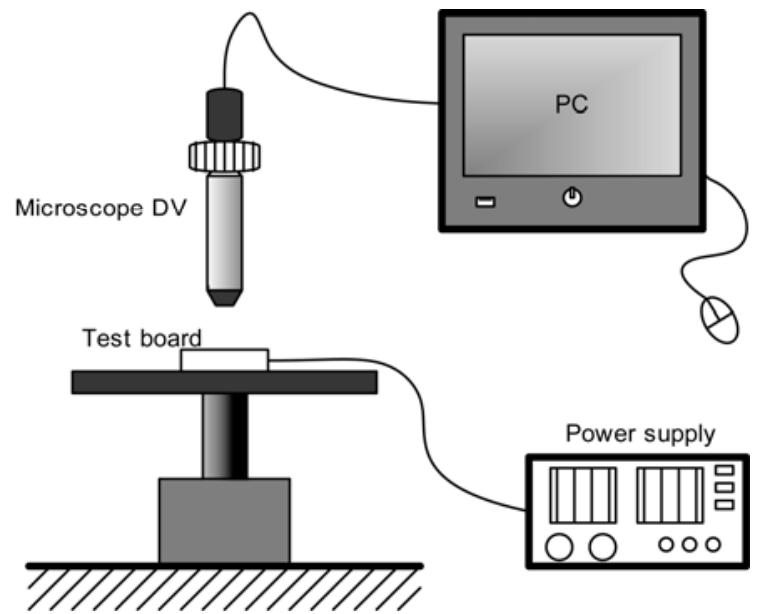

Figure 3 Experimental apparatus.

fill the square pits surrounded by steps, although the metal surface has a stronger wettability than the external photoresist. However, those electrolytic bubbles generated near the edges of steps on 1- $\mu \mathrm{m}$ specimens behave differently. At the beginning, the bubbles keep their centers on the side of the step's lower surface, as shown in Figure 4(a) and (b), indicating they are staying on the electrodes' surfaces. When big enough, each bubble center instantly moves to another side of the step edge, the upper surface of the step, as shown

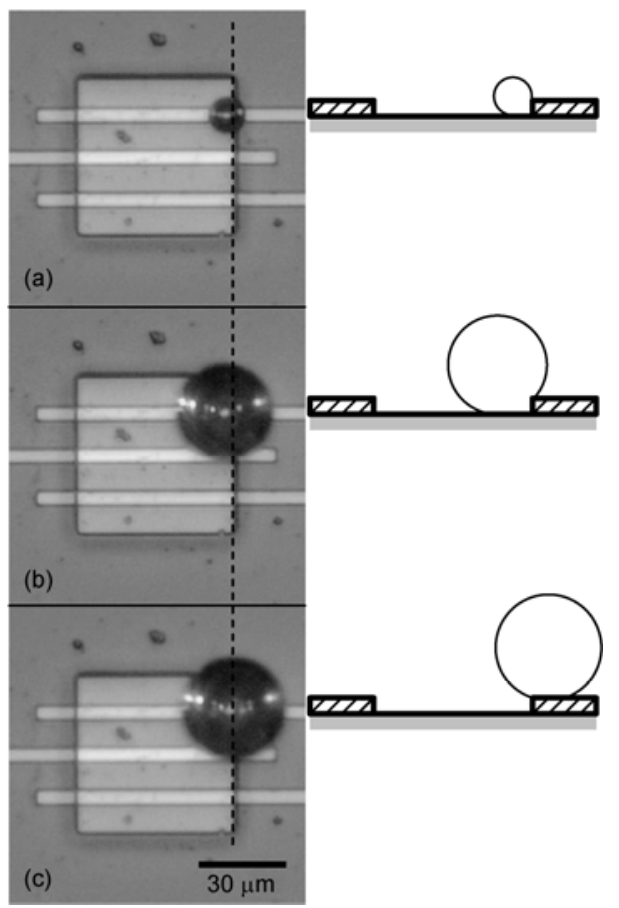

Figure 4 An electrolytic bubble generated on a specimen with a 1- $\mu \mathrm{m}$ step and its movement during growth. The dashed line indicates the step's position. (a) Frame recorded soon after the bubble is generated on the electrode surface; (b) frame recorded right before the movement, in which the bubble-center remains at the generation point;(c) frame recorded right after the movement, in which the bubble-center lies on the other side of the step edge. in Figure 4(c). Figure 4(b) and (c) shows the position of the same bubble in two neighboring frames, so the movement should finish within $40 \mathrm{~ms}$.

Because the photoresist used here has a weaker wettability and a bigger contact angle than the electrodes or the oxide layer, bubbles naturally have the potential to move towards the photoresist surface. In this situation, a step can act as a potential barrier to the movement, and therefore can constrain bubbles against the surface tension. However, for a particular bubble, a surface with a step much smaller than the scale of the bubble can be considered flat and smooth, and the constraining effect can be ignored. Conversely, for a particular step, a continuously growing bubble will eventually get big enough and result in failure of constraint. In the experiment, specimens with different thicknesses of photoresist layers perform quite differently, and instant movement just corresponds to failure. Failure of constraint means fault to correlative devices, so designers are faced with two issues: how to determine the bubble volume range for successful constraint, and how to determine the step parameters for a needed bubble volume. In the following sections, we used theoretical analysis and numerical simulation to study this failure and associated issues.

\section{Numerical simulation and analysis}

\subsection{Analytical model and method}

Structures used in the experiments can be simplified to a double-layer model, i.e. the substrate and the step layer, as illustrated in Figure 5. These two layers are of different materials, and this model is suitable for many situations in micro devices. The step height is $H$.

Both surfaces of the substrate and the step are ideally clean, inert and smooth, so the contact angles are determined by eq. (1). The contact angles are $\theta_{\mathrm{t}}$ and $\theta_{\mathrm{b}}$ for the materials of the step layer and substrate respectively.

On the micro scale, body forces, such as gravity and buoyancy, usually become much smaller than surface forces. Here it is reasonable to ignore the contributions of gravity and buoyancy, and presume that bubble shape is dominated by surface tension. Therefore, in the steady state, the internal and external pressure and bubble shape obey the Laplace equation

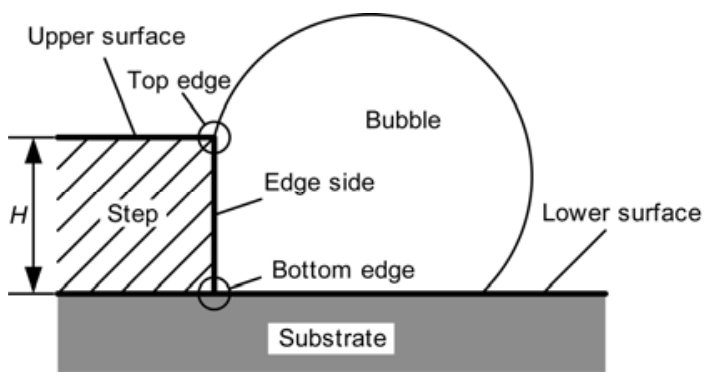

Figure 5 Step-shape structure and a constrained bubble. 


$$
p_{\text {in }}-p_{\text {out }}=\gamma_{\mathrm{LV}}\left(\frac{1}{R_{1}}+\frac{1}{R_{2}}\right),
$$

where $p_{\text {in }}$ and $p_{\text {out }}$ are the pressure inside and outside the bubble respectively, while $R_{1}$ and $R_{2}$ are two main curvature radii at any point on the gas-liquid interface. For a suspended static bubble, a spherical shape is the unique solution to eq. (2), and $R_{1}=R_{2}$. However, bubbles attaching to structures like step-shape structures, are more often non-spherical, and the two main curvature radii are more often different. For simplicity, we use only the 2-D model shown in Figure 5. The 2-D model physically corresponds to a gas column as in Figure 6, in which the contact angles on the plates at the two ends are exactly $90^{\circ}$. Though it does not match a real bubble, the 2-D model can reveal the reason and mechanism for the experimental phenomenon. In 2-D, bubbles degrade to 2-D round shape, and eq. (2) reduces to

$$
p_{\text {in }}-p_{\text {out }}=\gamma_{\mathrm{LV}} \frac{1}{R} \text {. }
$$

In the following simulation, we use the volume of fluid (VOF) method [18] to simulate the two-phase fluid flow, and use the continuum surface force (CSF) model [19] to introduce the surface forces. For the numerical simulation, we first place a bubble with a given volume at the bottom edge between the edge and lower surface, and then calculate the movement and deformation step by step until the bubble becomes steady.

\subsection{Numerical result for a basic case}

The first numerical simulations for a basic case use the conditions $\theta_{\mathrm{b}}=60^{\circ}, \theta_{\mathrm{t}}=120^{\circ}$ and $H=50 \mu \mathrm{m}$.

Results of simulations for small volumes, shown in Figure 7(a)-(d), indicate that bubbles can remain stably at the bottom edge of the step and appear as circular arcs, though various initial volumes result in different steady-state radii and various bubble shapes. In Figure 7(a), (b), where the bubble volumes are comparatively smaller, the steady-state bubble shapes are governed only by the contact angles on the edge side and the lower surface of the step, so these bubbles are of a similar shape. As the bubble volume

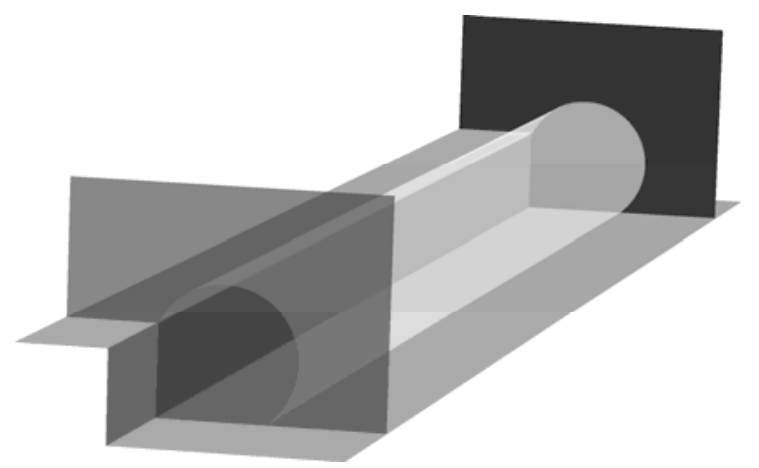

Figure 6 A gas column at the step edge, with the physical model corresponding to the 2-D bubble model. increases, the intersection of the gas-liquid interface and edge side moves upwards until it reaches the top edge. Thereafter, as the bubble volume goes on increasing, the intersection is pinned at the top edge, as in Figure 7(c), (d), until the angle $\phi$ increases to a critical angle.

Figure 8 shows the result for a slightly larger volume than that in Figure 7(d), revealing the dynamic process of the bubble movement. In Figure 8, the bubble can no longer remain stably at the bottom edge, but spontaneously moves
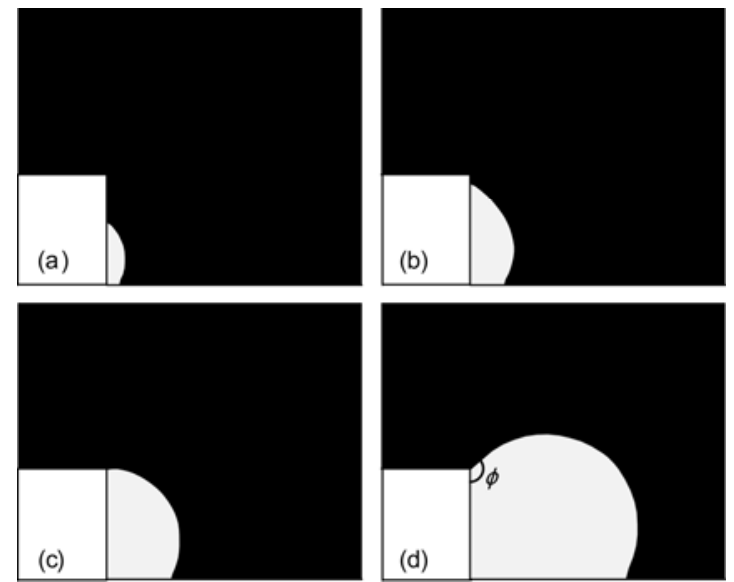

Figure 7 Numerical simulation for $\theta_{\mathrm{b}}=60^{\circ}$ and $\theta_{\mathrm{t}}=120^{\circ}$. Small bubbles hold stably at the bottom edge of the step.
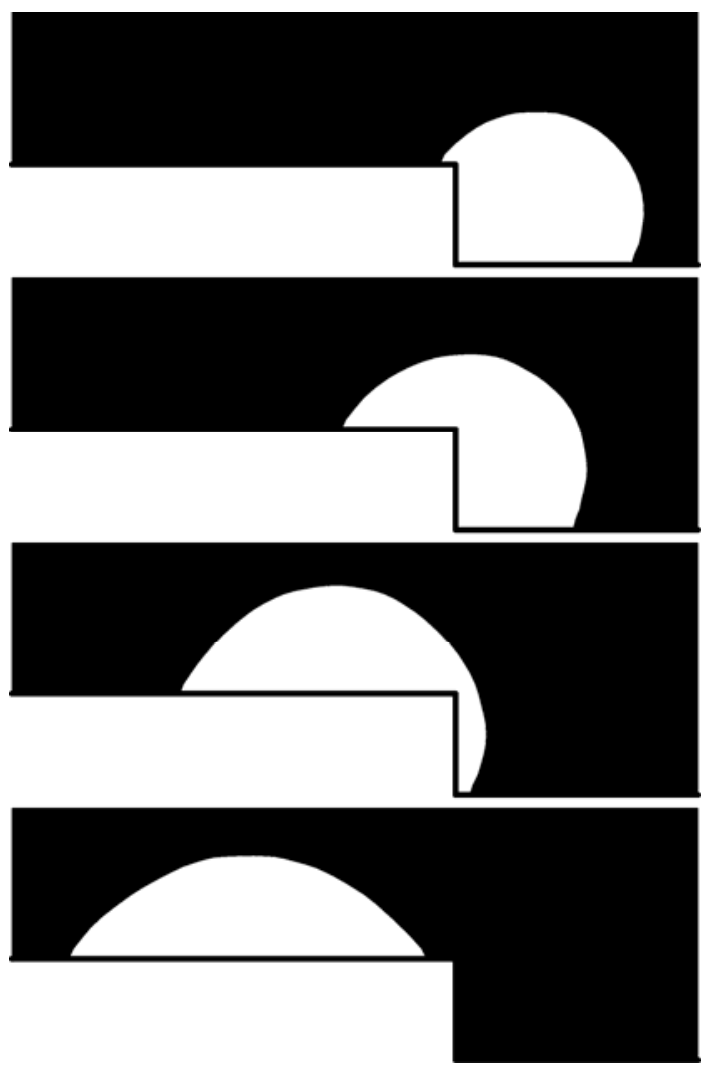

Figure 8 Numerical simulation for $\theta_{\mathrm{b}}=60^{\circ}$ and $\theta_{\mathrm{t}}=120^{\circ}$. A bubble larger than the critical volume moves onto the upper surface. 
onto the upper surface. This indicates a critical bubble volume $V_{\mathrm{C}}$, above which the bubble takes off from the lower surface and the constraint fails.

\subsection{Analysis and discussion}

Although the special case of $\theta_{\mathrm{b}}=60^{\circ}$ and $\theta_{\mathrm{t}}=120^{\circ}$ has been simulated above, the general conclusions can be derived from theoretical considerations.

For a bubble that remains stably at the step edge, as in Figure 7, its shape must meet two geometric conditions: First, because the gas-liquid interface is in equilibrium, the bubble must be in the shape of a circular arc according to eq. (3); second, the contact angles must be met, i.e. the angles between the gas-liquid interface and solid surface at the intersection must be equal to the contact angle $\theta_{\mathrm{b}}$ or $\theta_{\mathrm{t}}$, unless the intersection happens to join with a sharp edge as in Figure 7(c), (d). Figure 9 sketches the bubble growth process for Figure $7(\mathrm{c}),(\mathrm{d})$. At first, angle $\phi$ increases to gain the bubble volume until it reaches $270^{\circ}-\theta_{\mathrm{t}}$. Thereafter, angle $\phi$ can no longer increase because of the contact angle condition on the upper surface, and a tiny increment of the bubble volume will cause instability. Based on these geometric conditions, the bubble volume and curvature radius at the critical state can be analytically written as

$$
R_{\mathrm{C}}=\frac{H}{\cos \theta_{\mathrm{b}}-\cos \theta_{\mathrm{t}}}=\frac{1}{2} \frac{H}{\sin \left(\Theta^{+} / 2\right) \sin \left(\Theta^{-} / 2\right)}
$$

and

$$
V_{\mathrm{C}}=\frac{1}{2} R_{\mathrm{C}}{ }^{2}\left(\sin \Theta^{+}+\sin \Theta^{-}-\cos \Theta^{+} \sin \Theta^{-}+2 \pi-\Theta^{+}\right) L
$$

where $R_{\mathrm{C}}$ and $V_{\mathrm{C}}$ represent the critical radius and critical volume respectively, $L$ is the length of the gas column sketched in Figure 6, $H$ is the step height, and $\Theta^{+}=\theta_{\mathrm{t}}+\theta_{\mathrm{b}}$, $\Theta^{-}=\theta_{\mathrm{t}}-\theta_{\mathrm{b}}$.

To support the analysis, we use the VOF method and CSF model again to simulate bubble growth with a gas inlet at a low aeration speed of $0.01 \mathrm{~m} / \mathrm{s}$ at the bottom edge, and we record the critical bubble volume as soon as the bubble takes off from the lower surface. We simulate a series of cases in which $\theta_{\mathrm{b}}$ is fixed at $60^{\circ}$ and $\theta_{\mathrm{t}}$ is variable, and the numerical results show good consistency with the analytical solution as shown in Figure 10, though there are some

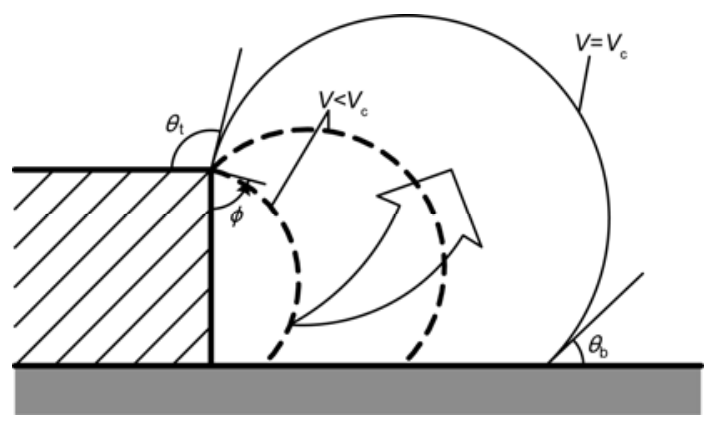

Figure 9 Sketch of the bubble growth process and critical state.

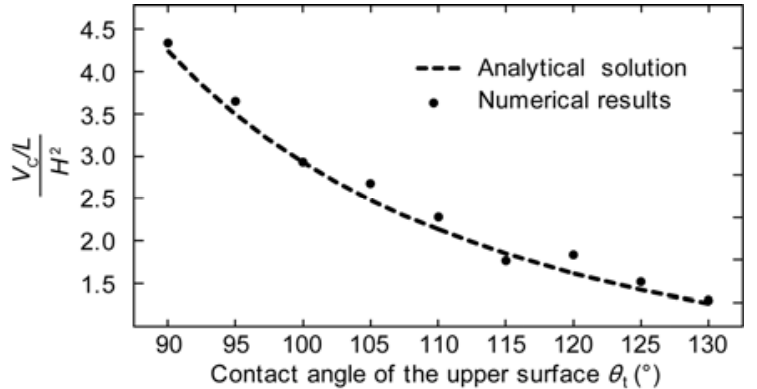

Figure 10 Critical bubble volumes obtained from numerical simulations with $\theta_{\mathrm{b}}=60^{\circ}$

fluctuations resulting presumably from numerical errors and the bubble expansion rate.

When $\cos \theta_{\mathrm{b}}-\cos \theta_{\mathrm{t}} \leqslant 0$, eq. (4) yields an unphysical value for $R_{\mathrm{C}}$, indicating no critical volume, and the bubble can stay indefinitely on the lower surface and grow rather big. Thus, the critical volume exists only when $\cos \theta_{\mathrm{b}}-\cos \theta_{\mathrm{t}}>0$, or $\theta_{\mathrm{b}}<\theta_{\mathrm{t}}$, that is, the wettability of the lower surface is stronger than that of the higher surface. In fact, when the higher surface has a weaker wettability, the bubble tends to be pulled on as mentioned above. However, the step plays a role as a barrier, and only those bubbles larger than the critical volume can overcome this barrier. This can explain the instant movement in the experiments above.

Furthermore, we can get a diagram of the critical bubble volume $\left(V_{\mathrm{C}} / H^{2} L\right)$ from eqs. (4) and (5) as shown in Figure 11 and the following criteria:

(i) If $\theta_{\mathrm{t}} \leqslant \theta_{\mathrm{b}}$, or below the $\infty$ line, the bubble can unconditionally stably remain at the bottom edge.

(ii) If $\theta_{\mathrm{t}}>\theta_{\mathrm{b}}$, or above the $\infty$ line, the bubble can stably remain when $V<V_{\mathrm{C}}$; otherwise, the bubble will move on.

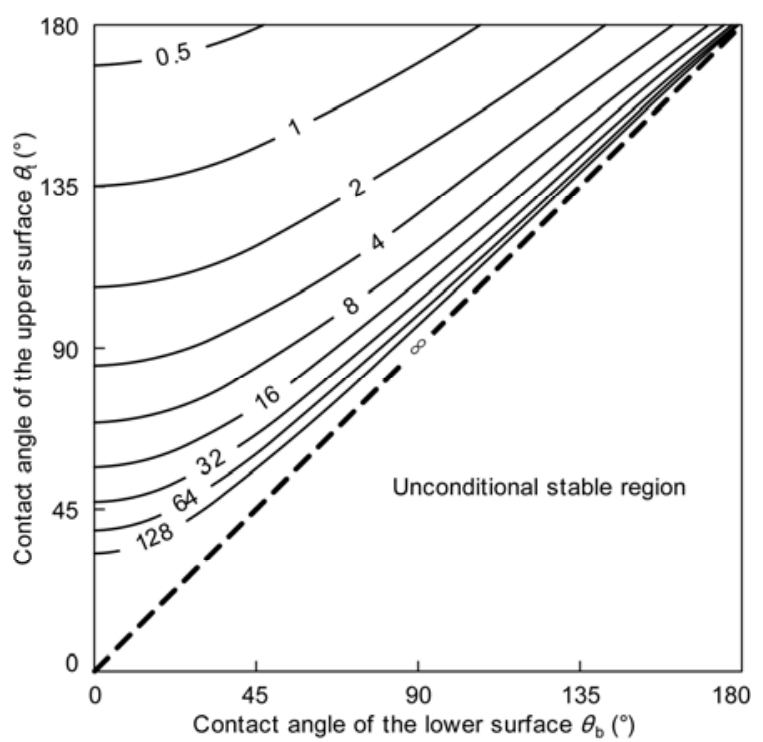

Figure 11 Contour map of the critical bubble volume $V_{\mathrm{C}} / H^{2} L$ for the 2-D model. Above the $\infty$ line, the bubble can stably hold at the bottom edge only when $V<V_{\mathrm{C}}$. Below the $\infty$ line, the bubble can unconditionally stably remain. 
According to the analysis above, the critical bubble volume can be determined by three factors: contact angles $\theta_{\mathrm{t}}, \theta_{\mathrm{b}}$ and the step height $H$, among which the step height is often easy to control during device design while the contact angles are usually limited by the materials and fabrication processes. Also, according to eqs. (4) and (5), when the contact angles are fixed, we can get $R_{\mathrm{C}} \propto H, V_{\mathrm{C}} \propto H^{2}$, so it is an alternative way to design a suitable step height to constrain bubbles with required volumes when we have few choices of materials or fabrication processes. Although there are differences between the simplified 2-D bubble model and a real 3-D bubble, similar critical volumes are expected for 3-D bubbles. Also, during device design, similar numerical methods can be used to solve a 3-D case for a more reliable critical volume. When needed, more detailed experiments can be carried out to draw diagrams for real bubbles.

\section{Conclusions}

Using experiments, theoretical analysis, and numerical simulation, we have studied the effect of step-shape structures in constraining bubbles and avoiding the migration caused by surface forces on the micro scale.

First, this constraining effect is confirmed by qualitative experimental observations on electrolysis bubbles. In these experiments, bubbles can stably remain at the step edges and adhere to the lower surfaces of the steps, though the upper surfaces have a weaker wettability. However, we also see constraint failure, that is, bubbles can gain enough volume to take off from the lower surface and move onto the upper surface.

Second, we studied constraint failure via numerical simulation and theoretical analysis based on a 2-D bubble model. This failure happens when the bubble expands over a critical volume, which has an analytical relationship with the contact angles of the step surfaces and the step height. We have presented the analytical relationship for the 2-D bubble model in this paper.

This work will be useful for designing correlative micro devices using constrained bubbles. Step-shape structures are a good choice when the wettabilities of device wall surfaces go against bubble constraint, and a suitable critical failure bubble volume can be determined beforehand by designing a proper step height.

This work was supported by the Specialized Research Fund for the Doctoral Program of Higher Education of China (20110002110077).

1 Zhang K, Jian A Q, Zhang X M, et al. Laser-induced thermal bubbles for microfluidic applications. Lab Chip, 2011, 11: 1389-1395

2 Ateya D A, Shah A A, Hua S Z. An electrolytically actuated micropump. Rev Sci Instrum, 2004, 75: 915-920

3 Ryu K, Chung S K, Cho S K. Micropumping by an acoustically excited oscillating bubble for automated implantable microfluidic devices. J Assoc Lab Automat, 2010, 15: 163-171

4 Chiu S H, Liu C H. An air-bubble-actuated micropump for on-chip blood transportation. Lab Chip, 2009, 9: 1524-1533

5 Tsou C, Huang C. Thermal bubble microfluidic gate based on SOI wafer. J MEMS, 2009, 18: 852-859

6 Son S U, Lee S S. Microfocusing using the thermal actuation of microbubbles. Microfluid Nanofluid, 2009, 6: 77-84

7 Mao X, Juluri B K, Lapsley M I, et al. Milliseconds microfluidic chaotic bubble mixer. Microfluid Nanofluid, 2010, 8: 139-144

8 Ahmed D, Mao X, Juluri B K, et al. A fast microfluidic mixer based on acoustically driven sidewall-trapped microbubbles. Microfluid Nanofluid, 2009, 7: 727-731

9 Ahmed D, Mao X, Shi J, et al. A millisecond micromixer via single-bubble-based acoustic streaming. Lab Chip, 2009, 9: 2738-2741

10 Kato H, Yamaguchi M. Enhancement of mixing by microbubble emission boiling in a microfluidic device. J Visualiz, 2009, 12: 267-274

11 Chan S C, Chen C R, Liu C H. A bubble-activated micropump with high-frequency flow reversal. Sens Actuators A, 2010, 163: 501-509

12 Liao K M, Chen R, Chou B C S. A novel thermal-bubble-based micromachined accelerometer. Sens Actuators A, 2006, 130: 282-289

13 Liao K M, Chen R. Novel two-dimensional micromachined accelerometer based on thermocapillary heat transfer. J Micro/Nanolith MEMS MOEMS, 2008, 7: 033011

14 Kang E, Lee D H, Kim C B, et al. A hemispherical microfluidic channel for the trapping and passive dissipation of microbubbles. $\mathrm{J}$ Micromech Microeng, 2010, 20: 045009

15 Gau H, Herminghaus S, Lenz P, et al. Liquid morphologies on structured surfaces: From microchannels to microchips. Science, 1999, 283: 46-49

16 Zhao B, Moore J S, Beebe D J. Surface-directed liquid flow inside microchannels. Science, 2001, 291: 1023-1026

17 Ling W Y L, Ng T W, Neild A. Effect of an encapsulated bubble in inhibiting droplet sliding. Langmuir, 2010, 26: 17695-17702

18 Hirt C W, Nichols B D. Volume of fluid (VOF) method for the dynamics of free boundaries. J Comput Phys, 1981, 39: 201-225

19 Brackbill J U, Kothe D B, Zemach C. A continuum method for modeling surface tension. J Comput Phys, 1992, 100: 335-354

Open Access This article is distributed under the terms of the Creative Commons Attribution License which permits any use, distribution, and reproduction in any medium, provided the original author(s) and source are credited. 